EPJ Web of Conferences 108, 02042 (2016)

DOI: $10.1051 /$ epjconf/201610802042

(C) Owned by the authors, published by EDP Sciences, 2016

\title{
Modeling of Intrinsic Josephson Junctions in High Temperature Superconductors under External Radiation in the Breakpoint Region
}

\author{
Yu. M. Shukrinov ${ }^{1,2, a}$, I. R. Rahmonov ${ }^{1,3, b}$, A. Plecenik ${ }^{4, c}$, O. I. Streltsova ${ }^{5, d}$, M. I. Zuev ${ }^{5, e}$, and \\ G. A. Ososkov ${ }^{5, f}$ \\ ${ }^{1}$ BLTP, Joint Institute for Nuclear Research, 141980 Dubna, Russia \\ ${ }^{2}$ Dubna International University for Nature, Society and Man, Dubna, Russia \\ ${ }^{3}$ Umarov Physical and Technical Institute, Dushanbe, Tajikistan \\ ${ }^{4}$ Department of Experimental Physics, Comenius University, Bratislava, Slovakia \\ ${ }^{5}$ LIT, Joint Institute for Nuclear Research, 141980 Dubna, Russia
}

\begin{abstract}
The current-voltage (IV) characteristics of the intrinsic Josephson junctions in high temperature superconductors under external electromagnetic radiation are calculated numerically in the parametric resonance region. We discuss a numerical method for calculation of the Shapiro step width on the amplitude of radiation. In order to accelerate computations we used parallelization by task parameter via Simple Linux Utility for Resource Management (SLURM) arrays and tested it in the case of a single junction. An analysis of the junction transitions between rotating and oscillating states in the branching region of IV-characteristics is presented.
\end{abstract}

\section{Introduction}

The high temperature superconductors show layered structures consisting of alternating superconducting (S-layers) and insulating layers. The S-layers form a system of intrinsic Josephson junctions (IJJ, JJs) [1]. Since the thickness of the S-layers is small enough (comparable to the Debye screening length), the electric charge is not screened inside the S-layers. The electric field between two S-layers (and the voltage in the formed JJs) depends on the corresponding values in the neighboring junctions. This is the consequence of the coupling between junctions $[2,3]$ which results in a specific modification of the behavior of the intrinsic JJs as compared to a single junction.

Shapiro steps at different resonance conditions demonstrate essential changes in the dependence of their width on the amplitude of the external radiation. Scaling of this dependence for shunted JJs was shown [4]. Another interesting problem is related to the effect of the external electromagnetic

\footnotetext{
a e-mail: shukrinv@theor.jinr.ru

be-mail: rahmonov@theor.jinr.ru,ilhom-tj@inbox.ru

ce-mail: plecenik@fmph.uniba.sk

$\mathrm{d}_{\mathrm{e}-\mathrm{mail}}$ strel@jinr.ru

e e-mail: zuevmax@jinr.ru

fe-mail: ososkov@jinr.ru
} 


\section{EPJ Web of Conferences}

radiation on the charge density waves stemming from the non-equilibrium in the intrinsic JJs. It was shown [5] that the Shapiro steps in the current-voltage (IV) characteristics result from the reaction of the system of coupled JJs to the external radiation as a whole.

In this paper, we analyze the effect of the external radiation on the IV-characteristics (IVC) of coupled Josephson junctions at small radiation amplitudes at a frequency corresponding to the parametric resonance in these systems. The amplitude dependence of the Shapiro steps width in the parametric resonance region unveiled by the numerical solutions is discussed.

\section{Numerical methods}

\subsection{IV-characteristics and charge in S-layers}

The dynamics of the IJJ in high temperature superconductors in the presence of electromagnetic radiation is described in the frame of the one-dimensional CCJJ+DC model [6] by the system of equations for the gauge-invariant phase differences $\varphi_{l}(t)$ between the $l$ and $(l+1)$-th S-layers

$$
\left\{\begin{array}{l}
\frac{\partial \varphi_{l}}{\partial t}=V_{l}-\alpha\left(V_{l+1}+V_{l-1}-2 V_{l}\right) \\
\frac{\partial V_{l}}{\partial t}=I-\sin \varphi_{l}-\beta \frac{\partial \varphi_{l}}{\partial t}+A \sin \omega t+I_{\text {noise }}
\end{array}\right.
$$

where $t$ is the dimensionless time normalized to the inverse plasma frequency $\omega_{p}^{-1}, \omega_{p}=\sqrt{2 e I_{c} / \hbar C}$, $\beta=1 / \sqrt{\beta_{c}}, \beta_{c}$ is the McCumber parameter, $\alpha$ gives the coupling between junctions, and $A$ is the amplitude of the radiation. To find the IVC of the IJJ stack, we solve the system of nonlinear secondorder differential equations (1) using a fourth order Runge-Kutta method. The voltage is measured in units of $V_{0}=\hbar \omega_{p} /(2 e)$, the frequency in units of $\omega_{p}$, the bias current $I$ and the amplitude of radiation $A$ in units of $I_{c}$.

A specific feature of the coupled JJs is the appearance of an electric charge in the S-layers which is calculated based on the Maxwell equation $\operatorname{div}\left(\varepsilon \varepsilon_{0} E\right)=Q$, where $\varepsilon$ and $\varepsilon_{0}$ are the relative dielectric and electric constants, respectively. The charge density $Q_{l}$ in the S-layer $l$ is proportional to the difference between the voltages $V_{l}$ and $V_{l+1}$ in the neighbour insulating layers $Q_{l}=Q_{0} \alpha\left(V_{l+1}-V_{l}\right)$, where $Q_{0}=\varepsilon \varepsilon_{0} V_{0} / r_{D}^{2}$. The appearance of the charge in S-layers results in specific phemonema related to the longitudinal plasma waves along the stack of the junctions. We have assumed an even number of junctions $N=10$ in the stack to escape the additional modulations of the electric charge oscillations in the parametric resonance region which appear in the case of an odd number of junctions [6] and to see clearly the effect of radiation only.

Numerical calculations were performed for a stack with a coupling parameter $\alpha=0.05$, a dissipation parameter $\beta=0.2$ and periodic boundary conditions. The amplitude of the external radiation $A$ is normalized to the value of the critical current $I_{c}$. At the chosen parameters of the Josephson junction and external radiation the difference between two Bessel maximums is around $15 A$ units. To demonstrate several maximums of these ossilations, the interval of changing $A$ in our simulations is $(0,45)$. The simulated IV-characteristics show a branch structure, each branch being determined by the number of junctions in the R-state (with rotating phase, $\left\langle d \varphi_{l} / d t\right\rangle \neq 0,\left\langle\sin \varphi_{l}\right\rangle=0$ ) and O-state (with oscillating phase, $\left\langle d \varphi_{l} / d t\right\rangle=0,\left\langle\sin \varphi_{l}\right\rangle \neq 0$ ) [7,8]. Details of the model and of the simulation procedure are described in [6]. 


\subsection{Amplitude dependence of the SS width in the case of a single JJ}

We develop a numerical method for the calculation of the Shapiro step width on the amplitude of the external radiation. For each value of the amplitude we simulate the current voltage characteristics and test at each step of the current the coincidence of the Josephson and radiation frequencies.

The results of numerical calculation of a single JJ IV-characteristic at $\beta=0.2$ under radiation with frequency $\omega=2$ and amplitude $A=0.5$ is presented in Fig. 1(a). It demonstrates the Shapiro step (SS) at $V=2$. The amplitude dependence of the SS width is shown in Fig. 1(b). The results of numerical calculations (large squares) agree with the theoretical dependence (small squares) based on the Bessel function [9].
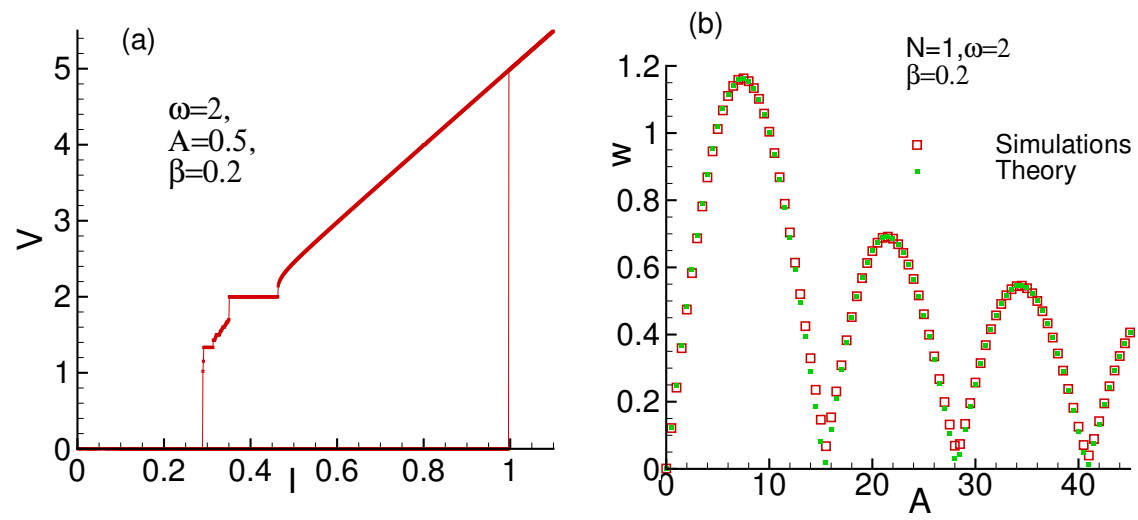

Figure 1. (a) IV-characteristic of JJ at $\beta=0.2$ under radiation with the frequency $\omega=2$ and amplitude $A=0.5$; (b) A-dependence of the Shapiro step (SS) width

\subsection{Parallel computing}

The task under solution possesses natural parallelism with respect to the data. In particular, the subtasks of finding the current voltage characteristic depending on the amplitude $A$ of the external radiation can be found independently. For example, the assessment of all characteristics of the amplitude value from $A_{\min }=0.0$ to $A_{\max }=45.0$ at a step of $\Delta A=0.225$, needs the solution of $N_{\text {job }}=201$ subtasks, an average computation time of $6 \mathrm{~min}$, hence an overall 20 hours for a sequential code.

Henceforth we discuss a parallel solution based on the distribution of computations by subtasks.

For computation distribution on the heterogeneous computing cluster HybriLIT of LIT JINR [10] the scheduling system SLURM (Simple Linux Utility for Resource Management) [11, 12] installed in the cluster was used. A mechanism of that system (job arrays) allows batch mode submitting and managing a large number of tasks $N_{\text {job }}$ carried out independently as a job array (jobs may be numbered from 0 to $N_{\mathrm{job}}-1$ with any step size or enumeration $(1,2,7, \ldots)$. It should be noted that each subtask of the generated array has an environment variable SLURM_ARRAY_TASK_ID that is installed according to the array index. That environment variable may be sent to the executable program to form the computed value that corresponds to its number.

Table 1 gives the results of computations carried out on the cluster HybriLIT. In the case of $N_{\text {job }}=$ 201, three computational nodes with 12-core Intel E5-2695 v2 processors were used in computations. We obtained a computation speedup of 122.8 . 
Table 1. Results of task computations according to the number of jobs

\begin{tabular}{|c|c|c|c|}
\hline $\begin{array}{c}\text { Number of } \\
\text { tasks } N_{\text {job }}\end{array}$ & $\begin{array}{c}\text { Max computational time } \\
\text { of one job (min) }\end{array}$ & $\begin{array}{c}\text { Time of sequential } \\
\text { computation (min) }\end{array}$ & Speedup \\
\cline { 1 - 3 } 21 & \multirow{2}{*}{6.3} & 77.5 & 12.3 \\
\cline { 1 - 3 } 201 & & 774 & 122.8 \\
\hline
\end{tabular}

\section{Coupled Josephson junctions: parametric resonance region features and branching of IV-characteristics}

The appearance of additional phenomena in a coupled system of JJs in comparison with a single junction such as the parametric resonance and the occurrence of longitudinal plasma waves along the c-axis (perpendicular to the S-layers) [13] adds specific complexity to the numerical calculations. The resonance properties are determined by the coupling and dissipation parameters, and also by the number of junctions in the stack. The coupling between the junctions leads to the branching of the IV-characteristics, and particularly, to a fine structure in the stacks with an even number of junctions while it is absent in the case of odd $N$. Irradiation with a frequency corresponding to the resonance region leads to a series of other interesting features [5, 14].
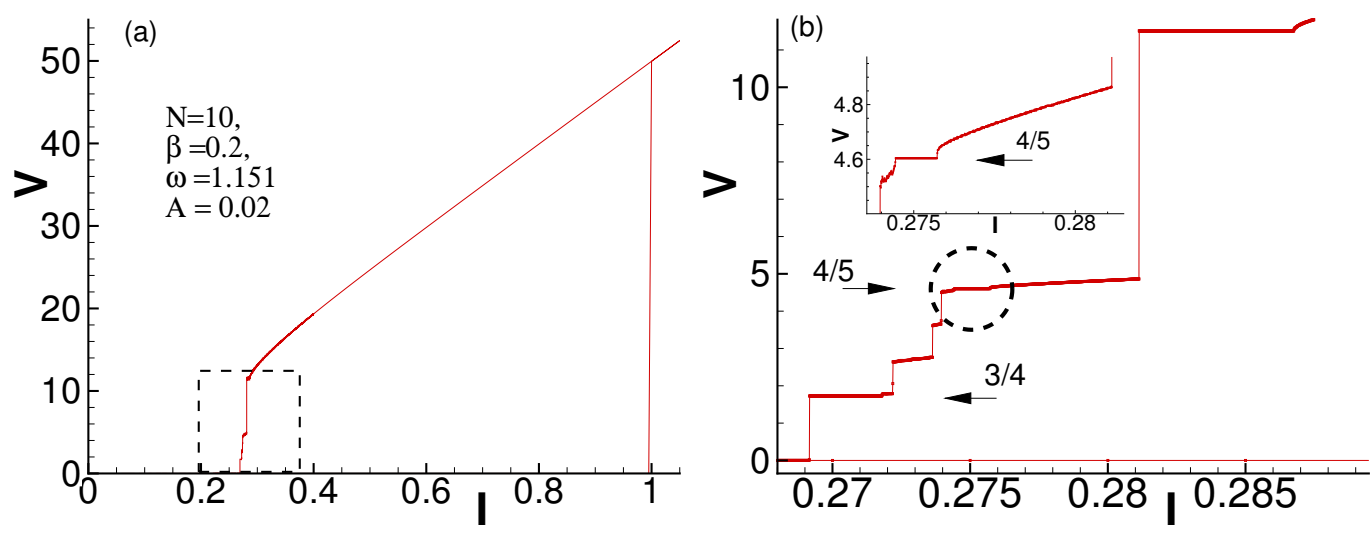

Figure 2. (a) IV characteristic of the stack with $10 \mathrm{JJs}$ under radiation with the frequency $\omega=1.151$ and amplitude $A=0.02$; (b) Enlarged part of IV characteristic is marked by the square in the left figure. Insets enlarge the parts marked by the circle.

In Fig. 2 we show the one-loop IV-characteristic of the stack with $10 \mathrm{JJs}$ under radiation with the frequency $\omega=1.151$ corresponding to the parametric resonance region and an enough small amplitude $A=0.02$. The IV-characteristic demonstrates a large hysteresis and branching. The branching part marked by the square is enlarged in the right figure. We also enlarge in the inset the part of branch marked by the circle which demonstrates steps in the current. The analysis of the steps indicates that we have obtained the Shapiro step subharmonics $3 / 4 \omega$ and $4 / 5 \omega$ on the inner branches. We note that they are a manifestation of second level subharmonics described by the continued fraction $N-1 / n$ with $N=1, n=4$ and $n=5$ [14].

To prove it, we plot in Fig. 3 the I-dependence of the average of $d \phi / d t$ for one junction in the same stack and at the same conditions as in Fig. 2. The frequencies $\omega=0.863$ and $\omega=0.921$ are resolved 


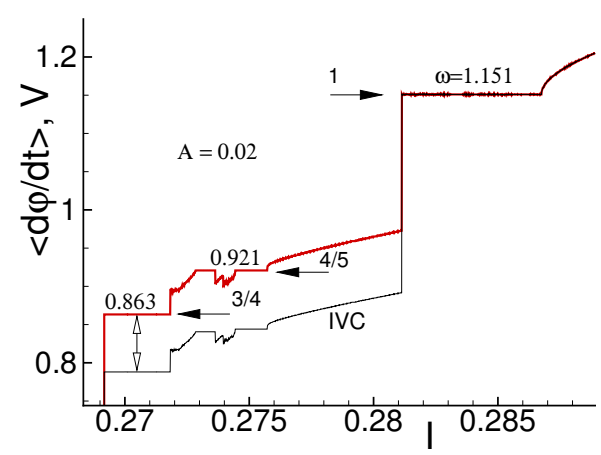

Figure 3. The I-dependence of the average of $d \varphi / d t$ for one $\mathrm{JJ}$ in the same stack and at the same conditions as in Fig.1. The thin line shows the IV-characteristic (IVC) of the same junction.

which are $3 / 4$ and $4 / 5$ parts of radiation frequency $\omega=1.151$, respectively. With the increase of $A$ the situation is changed. Thus, at $A=0.05$, all the observed steps in the IV-characteristic correspond to the resonance frequency only, even it they are related to the different inner branches. This fact stresses the specific Shapiro step behavior in the branching region of the IV-characteristic of the stack. The IV-characteristic in this figure also demonstrates that the SS voltage does not correspond to the related frequency (stressed by the double arrow). The origin of this difference is the voltage in the O-state junctions due to the coupling between the layers. Let us now discuss shortly the transitions from
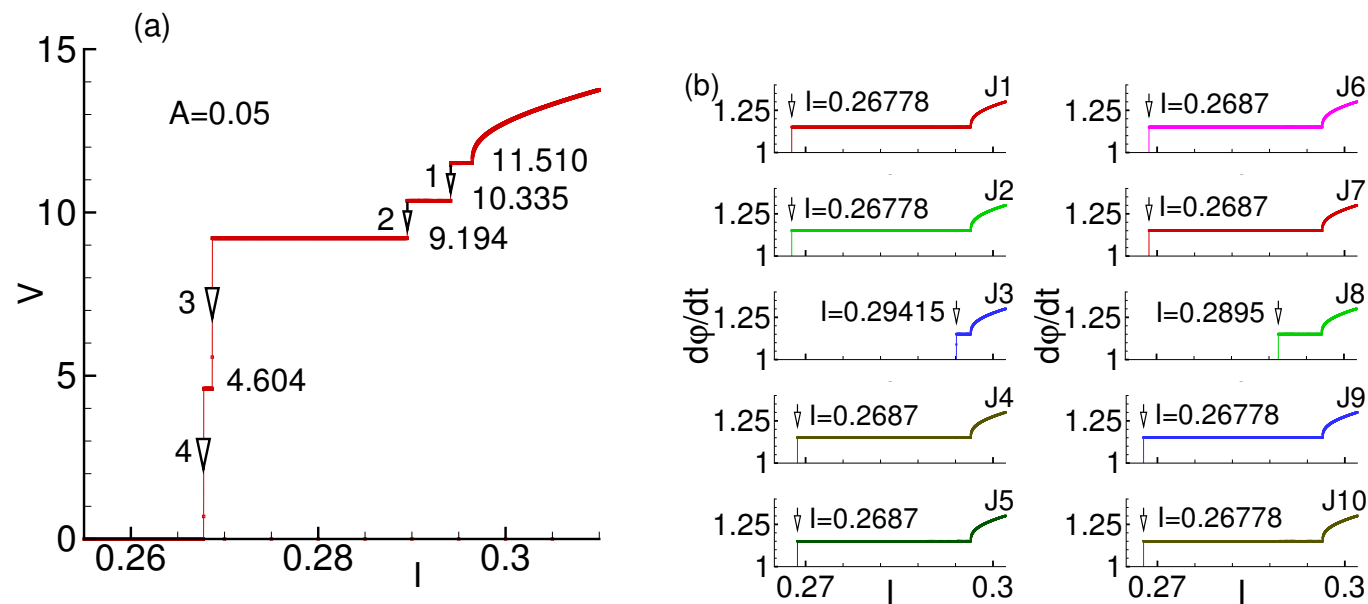

Figure 4. (a) Part of the IV-characteristic at $A=0.05$ in the branching region. The number near the step shows voltage value. Arrows with integers indicate transitions between different states of the stack. (b) Average derivative of the phase difference in each $\mathrm{JJ}$ in the stack as a function of the bias current at $A=0.05$.

rotating to oscillating states in JJs of the stack at some fixed values of the radiation amplitude. Figure 4 shows the part of the IV-characteristic at $A=0.05$, demonstrating a series of current steps and transitions between them with the decrease of the bias current. The first step at $V=11.51$ corresponds to SS on the outermost branch when all JJs are in the R-state. The index 1 reflects the transition when one $\mathrm{JJ}$ undergoes from R- to O-state, so the step at $V=10.335$ corresponds to the state R9O1 with $9 \mathrm{JJs}$ in the R-state and $1 \mathrm{JJ}$ in the O-state. Then the transition 2 happens which reflects a jump of 
another JJ from R- to O-state and so the step at $V=9.194$ corresponds to the state the $\mathrm{R} 8 \mathrm{O} 2$. The last step at $V=4.604$ is related to the state R4O6.

To test this analysis of JJs transitions and our interpretation of the observed steps, we show in Fig. 4(b) the average derivative of the phase difference at $A=0.05$ in each JJ of the stack as a function of the bias current. We see here the sequence of transitions at $I=0.29415$ (3-d JJ transition), $I=0.28950$ (8-th JJ), $I=0.26870$ (4-th, 5-th, 6-th, 7-th JJs) and $I=0.26778$ (the rest of JJs).

So in the case of the stack the numerical procedure for calculating the $A$-dependence of SS should be based on the simulation of the average derivative of the phase difference in each JJ in the stack. The described picture makes the program of numerical calculations of the amplitude dependence of the SS width in the IV-characteristics rather complex. Additional complexities should appear due to the possibility of chaotic behavior of the junctions and the noise in numerical simulations.

\section{Conclusions}

Specific features of the Shapiro steps in the branching region of the IV-characteristics of the coupled Josephson junctions have been resolved. These features should be taken into account in the development of an effective method for numerical calculation of the amplitude dependence of the SS width for stacks of coupled Josephson junctions.

\section{Acknowledgements}

This work is supported by the RFBR grant 15-29-01217 and a 2015 grant of the Plenipotentiary Representative of the Slovak Government at JINR.

Yu.M.S. and I.R.R. thank M. Maiti, S. Dubnička, M. Hnatič and J. Buša for discussions and support of this work.

\section{References}

[1] R. Kleiner, F. Steinmeyer, G. Kunkel, and P. Muller, Phys. Rev. Lett. 68, 2394 (1992)

[2] T. Koyama and M. Tachiki, Phys. Rev. B 54, 16183 (1996)

[3] S. N. Artemenko and A. G. Kobelkov, Phys. Rev. Lett. 78, 3551 (1997)

[4] Yu. M. Shukrinov, I. R. Rahmonov, K. V. Kulikov, P. Seidel, EPL 110, 47001 (2015)

[5] Yu. M. Shukrinov and H. Abdelhafiz, JETP Letters 98, 551 (2013).

[6] Y. M. Shukrinov, F.Mahfouzi, and N. F. Pedersen, Phys. Rev. B 75, 104508 (2007)

[7] H. Matsumoto, S. Sakamoto, F. Wajima, T. Koyama, M. Machida, Phys. Rev. B 60, 3666 (1999).

[8] Yu.M. Shukrinov, F. Mahfouzi, Physica C 434, 6 (2006).

[9] Likharev K. K., Dynamics of Josephson Junctions and Circuits (Gordon and Breach, New York, 1986)

[10] Heterogeneous computing cluster HybriLIT of LIT JINR. URL: http://hybrilit.jinr.ru

[11] The job management systems SLURM: Simple Linux utility for resource management. URL: http://slurm.schedmd.com/job_array.html

[12] Andy B. Yoo et al, LNCS 2862 (2003), pp 44-60.

[13] Yu. M. Shukrinov and F. Mahfouzi, Phys. Rev. Lett. 98, 157001 (2007)

[14] Y. M. Shukrinov, S. Y. Medvedeva, A. E. Botha, M. R. Kolahchi, and A. Irie, Phys. Rev. B 88, 214515 (2013)

[15] Yu. M. Shukrinov, I. R. Rahmonov, and M. A. Gaafar, Phys. Rev. B 86, 184502 (2012) 